\title{
Effets des variations climatiques à l'horizon 2050 sur la distribution phytogéographique de Tieghemella heckelii Pierre ex A. Chev. (Sapotaceae) en Côte d'Ivoire
}

\author{
Sopie Cybèle DOFFOU ${ }^{*}$, Kouassi KOUADIO ${ }^{1}$ et Hyppolite N'Da DIBI ${ }^{1,2}$ \\ ${ }^{1}$ UPR Botanique, Laboratoire des Milieux Naturels et Conservation de la Biodiversité, UFR Biosciences, \\ Université Félix Houphouët-Boigny, 22 BP 582 Abidjan 22, Côte d'Ivoire. \\ ${ }^{2}$ Centre Universitaire de Recherche Appliquée en Télédétection (CURAT), UFR Biosciences, Université Félix \\ Houphouët-Boigny, 22 BP 582 Abidjan 22, Côte d'Ivoire. \\ *Auteur correspondant ; E-mail : cybeledoffou@ gmail.com ; Tél : (+225) 07-07-23-56-20.
}

\begin{tabular}{lll}
\hline Received: 01-03-2021 & Accepted: 25-04-2021 & Published: 30-04-2021 \\
\hline
\end{tabular}

\section{RESUME}

Parmi les 36 espèces de bois d'œuvre inscrites sur la liste rouge de l'UICN, Tieghemella heckelii (Sapotaceae) ou Makoré est l'une des deux espèces qualifiées de danger d'extinction, à cause des activités anthropiques qui ont entrainé la réduction considérable des écosystèmes forestiers en Côte d'Ivoire. De plus, les variations climatiques influenceraient aussi l'aire de distribution de cette espèce menacée. D'où l'intérêt de cette étude dont l'objectif général vise à apporter des connaissances sur l'effet des variations climatiques sur la répartition phytogéographique de Makoré en Côte d'Ivoire, pour une gestion rationnelle de l'espèce. Il a été question de modéliser la distribution des aires favorables actuelles de Makoré dans les zones phytogéographiques; d'évaluer les effets potentiels des variations climatiques à l'horizon 2050 sur l'étendue des aires de distribution phytogéographique de Makoré et de déterminer les contributions des variables bioclimatiques importantes dans la modélisation de la distribution de Makoré. Pour ce faire, les coordonnées géographiques des spécimens de Makoré herborisés dans les trois herbiers nationaux et les individus inventoriés dans les zones phytogéographiques du pays, ont été recensées. Les scenarios RCP 6.0 et RCP 8.5 ont été utilisés pour la prédiction de la distribution de Makoré dans les conditions climatiques actuelles et futures. Les résultats des simulations montrent que les habitats favorables à la distribution de Makoré seront localisés préférentiellement dans les forêts denses humides sempervirentes du pays. L'importante contribution de la variable bioclimatique «précipitations du mois le plus sec (Bio 14) » signifie que la distribution future de Makoré sera principalement influencée par les variations de pluviométrie. Cette étude aidera certainement les gestionnaires du patrimoine forestier ivoirien dans leurs prises de décision sur la conservation et la protection de la biodiversité.

(C) 2021 International Formulae Group. All rights reserved.

Mots clés : Tieghemella heckelii (Sapotaceae), Aire de distribution, Modélisation, Variations bioclimatiques, Côte d'Ivoire. 


\title{
Effects of climatic variations in 2050 on the phytogeographic distribution of Tieghemella heckelii Pierre ex A. Chev. (Sapotaceae) in Côte d'Ivoire
}

\begin{abstract}
Among the 36 timber species listed on the IUCN Red List, Tieghemella heckelii (Sapotaceae) or Makoré is one of the two species classified as endangered, due to anthropogenic activities that have led to the considerable reduction of forest ecosystems in Côte d'Ivoire. In addition, climatic variations would also influence the distribution area of this threatened species. Hence the interest of this study whose general objective is to provide knowledge on the effect of climatic variations on the phytogeographic distribution of Makoré in Côte d'Ivoire, for a rational management of the species. The aim was to model the distribution of the current favourable areas of Makoré in the phytogeographical zones; to evaluate the potential effects of climatic variations in 2050 on the extent of the phytogeographical distribution of Makore and to determine the contributions of the important bioclimatic variables in the modelling of the distribution of Makoré. To do this, the geographical coordinates of Makore specimens in the three national herbaria and individuals inventoried in the country's phytogeographical zones were recorded. The RCP 6.0 and RCP 8.5 scenarios were used to predict the distribution of Makore under current and future climate conditions. The results of the simulations show that the habitats favourable to the distribution of Makoré will be located preferentially in the dense evergreen rainforests of the country. The significant contribution of the bioclimatic variable "rainfall in the driest month (Bio 14)" means that the future distribution of Makoré will be mainly influenced by rainfall variations. This study will certainly help the managers of the Ivorian forest heritage in their decision-making on the conservation and protection of biodiversity.
\end{abstract}

(C) 2021 International Formulae Group. All rights reserved.

Keywords: Tieghemella heckelii (Sapotaceae), Distribution area, Modelization, Bioclimatic variations, Côte d'Ivoire.

\section{INTRODUCTION}

La biodiversité qui est l'expression de la variété des êtres vivants et principalement sa composante végétale est reconnue comme un patrimoine vital commun à toute l'humanité (Manfo et al., 2015). En effet, l'homme dépend des plantes pour sa nourriture, son combustible, son bois, ses médicaments et bien d'autres services. Cependant, selon Konaté et Linsenmair (2010), cette composante végétale caractérisée, en majorité, par les écosystèmes forestiers et savanicoles font l'objet d'une agression sans précédente. En Côte d'Ivoire, les performances agricoles et l'exploitation forestière pour grumes, ont entrainé la destruction de plus de $83 \%$ des surfaces forestières. Ces activités humaines ont réduit le couvert végétal du pays, au point qu'à ce jour, les reliques des écosystèmes forestiers plus ou moins conservés sont les parcs nationaux, les forêts classées et les réserves naturelles (Adou Yao, 2005 ; Kouadio, 2007 ; Kouadio et al., 2012). Elles ont également occasionné la disparition ou la raréfaction de plusieurs espèces de plantes dont $53 \%$ des espèces de bois d'œuvre (Aké-Assi, 1997 ; Davo, 2011; Konan et al., 2015 ; UICN, 2019). C'est le cas de Tieghemella heckelii Pierre ex A. Chev. (Sapotaceae) ou Makoré. Cette espèce qui était anciennement abondante dans les forêts denses humides tropicales, est à ce jour, inscrite sur la liste rouge de l'UICN entant qu'une espèce en danger de disparition de la flore ivoirienne (UICN, 2019). Cependant, selon Aké-Assi (2002), malgré l'exploitation abusive dont ont été l'objet les arbres de valeur de Côte d'Ivoire au cours de ce dernier quart de siècle, on trouve encore quelques vieux Makoré atteignant des dimensions exceptionnelles (fût jusqu'à 40 mètres de hauteur et jusqu'à 2 mètres de diamètre, à la base). Cette essence forestière de grande valeur économique mérite d'être préservée pour une gestion rationnelle et durable. En plus de la pression d'origine anthropique sur les écosystèmes forestiers et savanicoles, s'ajoutent les fluctuations des 
variables climatiques. En effet, les variations des paramètres climatiques sont aujourd'hui reconnues comme l'une des principales menaces pour la survie des espèces et l'intégrité des écosystèmes partout dans le monde (Sala et al., 2001; Heller et Zavaleta, 2009). Selon IPCC (2007), les fluctuations des variables climatiques telles que les précipitations et la température ont une incidence directe sur la diversité biologique et sur la répartition géographique des habitats favorables aux espèces. La Côte d'ivoire, à l'instar des pays africains, subit aussi les impacts du changement climatique sur la biodiversité, selon les travaux de recherches de Kouakou et al. (2012), Sorokoby et al. (2013), Kouamé et al. (2014), Oga et al. (2016). Ces auteurs prédisent même une augmentation de la température et une baisse des précipitations sur la majeure partie du pays. C'est dans ce sens que quelques études récentes aient été réalisées. Il s'agit entre autres de l'impact des changements climatiques sur la distribution des Epiphytes (Gnagbo et al., 2015), des Rubiaceae (Tuo et al., 2017), des espèces endémiques en Côte d'Ivoire (Vroh et al., 2016), des espèces végétales consommées par les chimpanzés dans le Parc national de Taï (Condé, 2017) et de certaines Acanthaceae en Côte d'Ivoire (Asseh, 2019 ; Asseh et al., 2019). Cependant, celle relative à l'effet des conditions climatiques futures sur l'aire de distribution de Makoré reste encore à documenter. D'où l'intérêt de cette étude qui a pour objectif général de disposer des données sur l'effet des variations climatiques sur la répartition phytogéographique de Makoré en Côte d'Ivoire, pour une gestion rationnelle des individus de l'espèce encore existants. Il a s'agit de :

- modéliser la distribution des aires favorables actuelles de Makoré dans les zones phytogéoraphiques;

- évaluer les effets potentiels des variations climatiques à l'horizon 2050 sur l'étendue des aires de distribution phytogéographique de Makoré, à l'aide des scenarios RCP 6.0 et RCP 8.5;

- déterminer les contributions des variables bioclimatiques importantes dans la modélisation de la distribution de Makoré.

\section{MATERIEL ET METHODES Site d'étude}

La présente étude a été réalisée en Côte d'Ivoire (Afrique de l'ouest) qui s'inscrit dans un carré dont les côtés sont localisés, d'une part, entre $4^{\circ} 30$ et $10^{\circ} 30$ de latitude Nord et, d'autre part, entre $2^{\circ} 30$ et $8^{\circ} 30$ de longitude Ouest, avec une superficie estimée à $322,500 \mathrm{Km}^{2}$. L'étude couvre quatre zones climatiques : le climat subéquatorial ; le climat tropical humide; le climat tropical et le climat de montagne. La végétation de la Côte d'Ivoire est subdivisée en deux grands domaines qui renferment les formations végétales dominantes. Le domaine guinéen comprend : la végétation littorale, la forêt dense humide sempervirente, la forêt dense humide semi-décidue, la forêt de montagne et la végétation de transition forêtsavane. Quant au domaine soudanais, il renferme les savanes arbustives et arborées, les forêts claires, les galeries forestières (Figure 1).

Le matériel biologique utilisé, au cours de cette étude, est composé de planches d'herbier et des individus de Makoré inventoriés dans les différentes zones phytogéographiques de la Côte d'Ivoire, notamment, dans les forêts classées, les parcs nationaux et réserves et dans les jardins botaniques du Centre National de Floristique et de Bingerville et aussi dans les plantations agricoles. Le logiciel Maxent a servi à la modélisation de l'aire de distribution de Makoré. Pour la cartographie de la distribution géographique des habitats favorables pour les périodes climatiques actuelle et futur, le logiciel QGIS 3.12 a été utilisé.

\section{Collecte de données}

Pour la collecte des données sur les occurrences de Makoré, trois herbiers de Côte d'Ivoire ont été consultés. Il s'agit de l'Herbier National de Côte d'Ivoire logé au Centre National de Floristique (CNF) de l'Université Félix HOUPHOUËT-BOIGNY, de l'Herbier de l'Institut Botanique Aké-Assi (IBAAN) situé à Yopougon-Anonkoi et de l'herbier du Centre Suisse de Recherche Scientifique (CSRS) situé à Adiopodoumé. Tous les spécimens de Makoré herborisés ont été recensés. Pour chaque planche d'herbier consultée, les informations portant les coordonnées géographiques ont été notés. Des 
informations additionnelles relatives à l'inventaire de Makoré dans certaines localités du pays ont été obtenues à partir de la base de données du GBIF (Global Biodiversity Information Facility) et dans des articles, des livres et des thèses. Pour certaines planches d'herbier dont les coordonnées géographiques (longitude et latitude) sont manquantes, elles ont été corrigées à partir des données extraites dans le Gazettiers de Côte d'Ivoire. Pour la modélisation de la distribution de Makoré en Côte d'Ivoire, dix-neuf (19) variables bioclimatiques consignées dans le Tableau 1 ont été extraites à partir des bases de données du Worldclim version 1.4 via le site www.worldbioclim.org avec une résolution de 2,5 arc-minute au sol et de données de CCAFS (www.ccafs-climate.org).

\section{Choix des scénarios pour la modélisation de l'aire de distribution de Makoré}

Pour les simulations climatiques futures à l'horizon 2050, il existe plusieurs scénarios issus du rapport spécial du Groupe d'Experts Intergouvernemental sur l'Evolution du Climat (GIEC) sur les scénarios d'émission (SRES). Un scénario est une description vraisemblable, cohérente et intrinsèquement homogène d'un état futur du climat du globe (GIEC, 2007 ; 2013). Les changements moyens de températures et des précipitations se traduisent aussi par des changements dans divers types d'événements climatiques dont la probabilité d'occurrence est plus ou moins bien connue. Ainsi, Kouakou (2014) a indiqué que l'un des scénarios élaborés pour estimer l'ampleur des changements climatiques futurs (horizon 2050) est le Representative Concentration Pathways version 4.5 (RCP 4.5) et prédit une hausse de la température de $3{ }^{\circ} \mathrm{C}$ d'ici 2100 sur la majeure partie de la Côte d'Ivoire allant du nord au sud. Pour la pluviométrie, le même scénario utilisé indique une baisse journalière de $8 \%$ des précipitations, durant les cent prochaines années. Pour cette étude deux (02) scénarios ont été utilisés; il s'agit de RCP 6.0 (estimation dite «modérée » de l'amplification des changements climatiques futurs (horizon 2050)) et de RCP 8.5 (estimation dite «forte» de l'amplification des changements climatiques futurs (horizon 2050)).

La modélisation de la distribution de Makoré en Côte d'Ivoire s'est faite à partir du logiciel Maximum Entropie (MaxEnt), comme utilisé par Asseh (2019) et Asseh et al. (2019) au cours de leurs travaux sur la modélisation de l'aire de distribution de quelques espèces des Acanthaceae. Selon Phillips et al. (2006), c'est un programme qui permet d'estimer et de prédire la distribution spatiale d'une espèce à partir des données de présence de l'espèce en relation avec les conditions environnementales dans la zone d'intérêt.

\section{Modélisation de la distribution de Makoré}

Les 19 variables bioclimatiques actuelles et futures (Tableau 1) retenues pour la modélisation de la distribution de Makoré ont été soumises à un test de corrélation. Ce test qui met en évidence les liens existants entre les variables, a permis de sélectionner les variables les moins corrélées (coefficient de corrélation inférieur en valeur absolue à 0,8). Pour Elith et al. (2011), une forte corrélation entre les variables entraine un biais sur les prédictions. La modélisation s'est faite en important dans l'algorithme de traitement du logiciel MaxEnt les coordonnées géographiques des 85 occurrences de Makoré collectées et qui ont été enregistrées dans un fichier au format CSV, selon la méthode de Gbesso et al. (2013). Par la suite, les 19 variables bioclimatiques actuelles et futures (horizon 2050), consignées dans le Tableau 1, ont été intégrées dans le même algorithme, afin de déterminer leurs contributions, en fonction des scénarios de simulation (RCP 6.0 et RCP. 8.5). Enfin, un test de Jackknife a été effectué sur ces variables bioclimatiques considérées pour déterminer celles qui contribuent le plus à la modélisation, comme l'a recommandé Fandohan et al. (2013).

\section{Analyse des données et validation du processus de modélisation}

Les différents niveaux d'habitats de Makoré ont été classifiés à partir des seuils de probabilités logistiques de présence générée par MaXent. La probabilité logistique d'occurrences d'une espèce est considérée comme indicatrice de la qualité des habitats 
pour l'espèce et elle varie entre 0 et 1 (Liu et al., 2013). Pour une valeur de probabilité inférieure à 0,229, l'habitat est dit "potentiellement non favorable" ; l'habitat est dit "potentiellement favorable" pour les valeurs supérieures à 0,229. L'étendue de chaque niveau d'habitat (aire et pourcentage) aussi bien sous les conditions actuelles que futures a été estimée en vue d'évaluer le gain ou la perte dans l'aire potentiellement favorable au Makoré à l'échelle de la Côte d'Ivoire. Cette estimation s'est effectué à partir de l'outil «spatiale analyst » du logiciel QGIS dans sa version 3.12. Selon Toyi et al. (2013), les taux de changement (Tc) des habitats "potentiellement favorables" dans le présent en habitats "potentiellement non favorables" dans le futur et vice versa ont été estimés pour chaque modèle climatique à partir de la formule suivante :

$$
\mathrm{Tc}=((\mathrm{A} 2-\mathrm{A} 1) / \mathrm{A} 1) \times 100
$$

A1 et A2 sont respectivement les superficies initiales (condition climatique présente) et finales (condition climatique future) de présence de l'espèce. Les valeurs positives de Tc indiquent des gains de superficie de présence tandis que les valeurs négatives traduisent les pertes de superficie de l'espèce. Dans cette étude, deux types d'habitat ont été considérés dans la période futur (horizon 2050) : les habitats "favorables" et les habitats "très favorables", et le calcul des superficies initiales et finales a été fait, en fonction des deux scénarios de simulation (RCP 6.0 et RCP 8.5).

$\mathrm{La}$ contribution des variables bioclimatiques a été analysée aussi, en fonction des conditions climatiques et des deux scénarios. Dans cette étude, les valeurs de contribution supérieures à $10 \%$ de ces variables ont été considérées. Concernant la fiabilité de la modélisation de l'aire de distribution de Makoré, la statistique AUC (Area Under the Curve) a été utlisée, selon les recommandations de Phillips et al. (2006). Pour l'interprétation de l'AUC, les travaux de Araújo et al. (2005) ont servi de référence (Tableau 2). Les valeurs de l'AUC ont été considérées en en fonction des conditions climatiques et des deux scénarios.

Tableau 1: Variables climatiques utilisées pour la modélisation de la distribution géographique de Makoré.

\begin{tabular}{ll}
\hline Codes & Dénomination des variables bioclimatiques \\
\hline BIO1 & Température moyenne annuelle \\
BIO2 & Étendue diurne moyenne (Moyenne mensuelle de (tmax - tmin) \\
BIO3 & Isothermalité (BIO2/BIO7) $(* 100)$ \\
BIO4 & Saisonnalité des températures (écart-type $* 100)$ \\
BIO5 & Température maximale du mois le plus chaud \\
BIO6 & Température minimale du mois le plus frais \\
BIO7 & Étendue annuelle des températures (BIO5-BIO6) \\
BIO8 & Température moyenne du trimestre le plus arrosé \\
BIO9 & Température moyenne du trimestre le plus sec \\
BIO10 & Température moyenne du trimestre le plus chaud \\
BIO11 & Température moyenne du trimestre le plus frais \\
BIO12 & Précipitations annuelles \\
BIO13 & Précipitations du mois le plus arrosé \\
BIO14 & Précipitations du mois le plus sec \\
BIO15 & Saisonnalité des précipitations (coefficient de variation) \\
BIO16 & Précipitations du trimestre le plus arrosé \\
BIO17 & Précipitations du trimestre le plus sec \\
BIO18 & Précipitations du trimestre le plus chaud \\
BIO19 & Précipitations du trimestre le plus frais \\
\hline
\end{tabular}




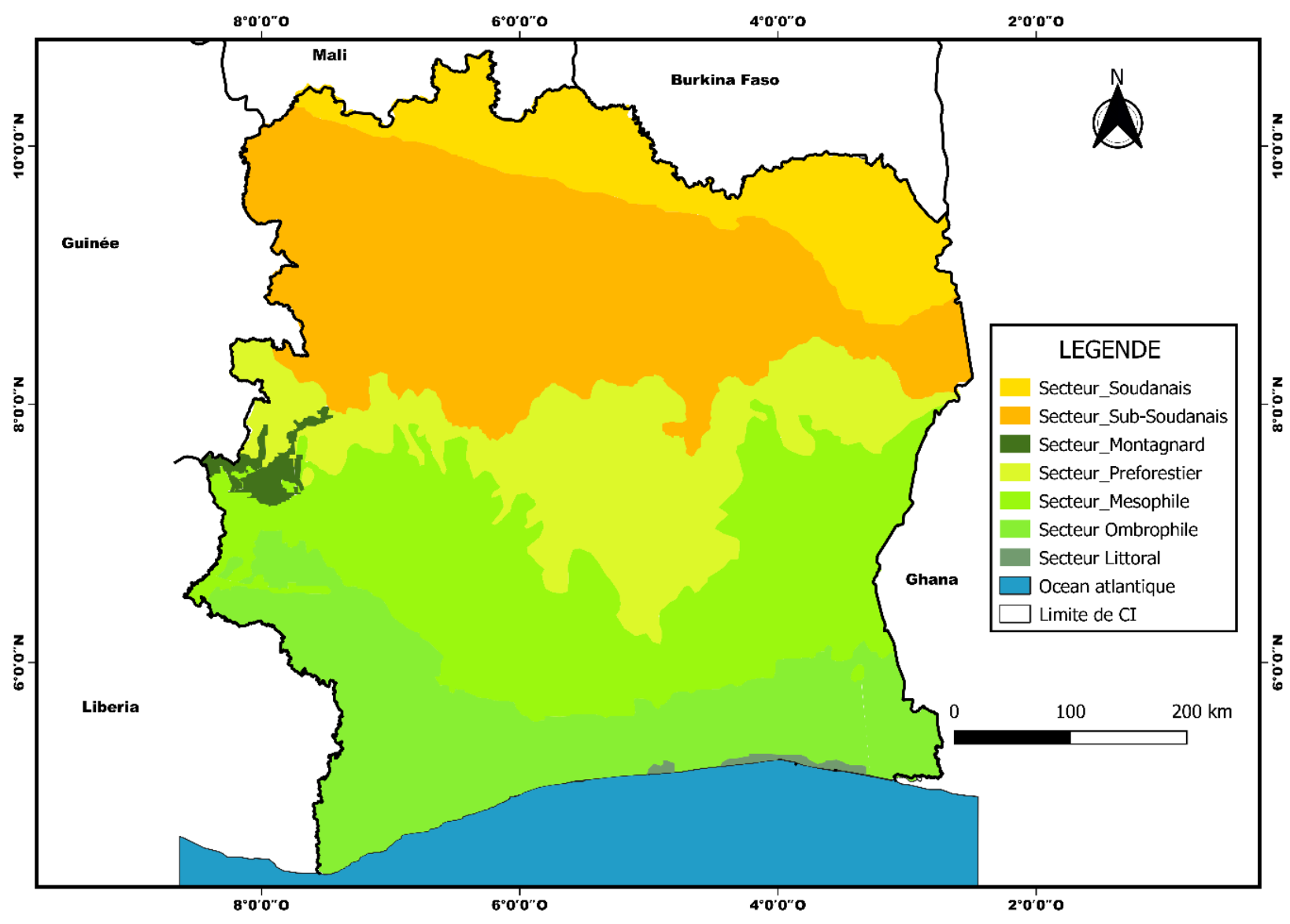

Figure 1 : Carte des types de végétations des domaines guinéen et soudanais de la Côte d'Ivoire. (Source: Brou, 2005).

Tableau 2: Evaluation de la fiabilité de la modélisation de la distribution phytogéographique de Makoré à travers l'interprétation des valeurs de l'AUC.

\begin{tabular}{cc}
\hline Valeurs de l'AUC & Interprétation de l'AUC \\
\hline $1,00>$ AUC $>0,90$ & Excellente \\
$0,80<$ AUC $<0,90$ & Bonne \\
$0,70<$ AUC $<0,80$ & Acceptable \\
$0,60<$ AUC $<0,70$ & Mauvaise \\
$0,50<$ AUC $<0,60$ & Invalide \\
\hline AUC : Area Under the Curve
\end{tabular}




\section{RESULTATS}

\section{Distribution des habitats favorables de Makoré pour les périodes présent et futur à l'aide du RCP 6.0}

Les résultats des différentes simulations montrent que pour la période actuelle les habitats "favorables" de Makoré couvrent environ $12,70 \%$ du territoire ivoirien, soit $40741 \mathrm{~km}^{2}$ de superficie (Tableau 3). Cette zone favorable est située en grande partie dans l'Ouest montagneux de la Côte d'Ivoire, faiblement sur le littoral et dans l'Est et moyennement dans le Sud du pays (Figure 2). Pour les habitats "très favorable", ils occupent environ 9,98\% de la couverture végétale, soit $32031 \mathrm{~km}^{2}$ de superficie (Tableau 3). Ce type d'habitat de Makoré est localisé préférentiellement dans le Sud-Est du pays, presque sur tout le long du littoral et dans le Sud-Ouest. Quel que soit le type d'habitats ("favorables" ou "très favorable"), l'aire de distribution de Makoré se situe dans la forêt dense humide sempervirente et le littoral du domaine guinéen (Figure 2). Pour la période future (horizon 2050), les prédictions des variables bioclimatiques indiquent une perte d'habitat de $4779 \mathrm{~km}^{2}$ des zones "favorables" et un gain de $4995 \mathrm{~km}^{2}$ pour les zones "très favorable" (Tableau 3 ).

\section{Distribution des habitats favorables de Makoré pour les périodes présent et futur à l'aide du RCP 8.5}

Les prédictions obtenues avec le scénario RCP 6.0 pour la distribution de Makoré dans les zones phytogéographiques de la Côte d'Ivoire sont identiques avec le scénario RCP 8.5, dans les conditions climatiques actuelles. Pour les conditions climatiques futures (horizon 2050), les simulations montrent que les habitats "favorables" occupent $35949 \mathrm{~km}^{2}$ de superficie, soit $11,15 \%$ du territoire national tandis que les habitats "très favorables" couvrent $36005 \mathrm{~km}^{2}$ de superficie, soit
$11,17 \%$ du total de la couverture végétale du pays (Tableau 4). L'aire de distribution de Makoré pour les zones "favorables" est située principalement dans l'Ouest en décrivant un "V" avec la pointe ouverte dans le Sud-littoral et s'étend dans l'est du pays (Figure 3). Pour les zones "très favorables", elles sont potentiellement localisées dans le Sud-Est, le Sud-Littoral et une poche dans la partie montagneuse de la Côte d'Ivoire (Figure 3). Les différentes proportions de superficie occupées par les deux types d'habitats dans les conditions climatiques futures (horizon 2050) indiquent une réduction d'aire de distribution de Makoré pour les habitats "favorables" et un gain de superficie pour les habitats "très favorables" par rapport aux conditions climatiques actuelles.

\section{Evaluation des tendances évolutives de l'aire de distribution de Makoré}

En comparant les prédictions de la distribution de Makoré des conditions climatiques futures (horizon 2050) à celles de la période actuelle, la Figure 4 montre que les habitats "non favorables" au Makoré sont de l'ordre de $77,46 \%$ du territoire ivoirien. Ces milieux écologiques concernent la forêt dense humide semi-décidue, la zone de transition forêt-savane dans le domaine guinéen et tout le domaine soudanais. S'agissant de la conversion des habitats, les simulations des scénarios RCP 6.0 et RCP 8.5 indiquent une perte des habitats de l'ordre de $1,55 \%$ dans les zones potentiellement "favorables" alors que dans les milieux écologiques potentiellement "très favorables", l'on note un gain de superficie de l'ordre de 1,35\% (Figure 4).

\section{Contributions des variables bioclimatiques et validation des scénarios pour la modélisation de la distribution de Makoré}

L'analyse des résultats du Tableau 5 a permis de sélectionner au total quatorze (14) variables bioclimatiques moins corrélées, en 
fonction des scénarios. Il s'agit pour la période actuelle de onze (11) variables bioclimatique (Tableau 5) avec la variable bioclimatique $\mathrm{BIO} 14$ présentant la plus forte valeur de contribution qui est de 53,8\%, suivie de BIO16 avec 12,3\% (Tableau 5), pour la modélisation de la distribution géographique de Makoré.

Pour la période future (horizon 2050) avec le scénario RCP 6.0, dix (10) variables bioclimatiques contributrices ont été retenues (Tableau 5). Pour ce scénario, la plus forte contribution a été enregistrée chez BIO14 avec la valeur de $47,7 \%$. Viennent en deuxième et troisième position $\mathrm{BIO} 13$ et BIO2 avec respectivement 14,7 et $13,6 \%$ de contributions (Tableau 5). Concernant le scénario RCP 8.5 , on note dix (10) variables bioclimatiques (Tableau 5) et la plus forte contribution obtenue est $45,7 \%$ pour $\mathrm{BIO} 14$ qui est suivie de BIO13 avec 24,7\% (Tableau 5).

Le scénario RCP 6.0 a généré des valeurs de l'AUC qui varient de 0,905 à 0,939 pour la période actuelle et de 0,904 à 0,945 pour la période future (horizon 2050). Pour le scénario RCP 8.5, les valeurs de l'AUC sont de 0,901 à 0,941 pour la période future (horizon 2050) alors que celles de la période actuelle sont identiques aux valeurs générées par le scénario RCP 6.0.

Tableau 3 : Variation de la superficie et de la tendance de l'aire de distribution de Makoré en fonction des périodes, à l'aide du scénario RCP 6.0.

\begin{tabular}{lcccccc}
\hline & \multicolumn{5}{c}{ Aire de distribution } \\
\cline { 2 - 7 } Périodes & \multicolumn{7}{c}{ Zones non favorable } & \multicolumn{2}{c}{ Zones favorable } & \multicolumn{2}{c}{ Zones très favorables } \\
\cline { 2 - 7 } & $\begin{array}{c}\text { Superficie } \\
\left(\mathrm{Km}^{2}\right)\end{array}$ & $\begin{array}{c}\text { Tendance } \\
(\%)\end{array}$ & $\begin{array}{c}\text { Superficie } \\
\left(\mathrm{Km}^{2}\right)\end{array}$ & $\begin{array}{c}\text { Tendance } \\
(\%)\end{array}$ & $\begin{array}{c}\text { Superficie } \\
\left(\mathrm{Km}^{2}\right)\end{array}$ & $\begin{array}{c}\text { Tendance } \\
(\%)\end{array}$ \\
Actuelle & 249263 & 77,32 & 40741 & 12,70 & 32031 & 9,98 \\
$\begin{array}{l}\text { Future } \\
(\mathbf{2 0 5 0 )}\end{array}$ & 249414 & 77,36 & 35962 & 11,15 & 37026 & 11,48 \\
& & & $\mathbf{- 4 ~ 7 7 9}$ & $\mathbf{- 1 , 5 5}$ & $\mathbf{+ 4 ~ 9 9 5}$ & $\mathbf{+ 1 , 5 0}$ \\
\hline
\end{tabular}

Le signe (-) indique une perte d'habitat favorable et le signe (+) indique un gain.

RCP : Representative Concentration Pathways (scénario élaboré pour stimuler l'ampleur des changements climatiques futurs). 

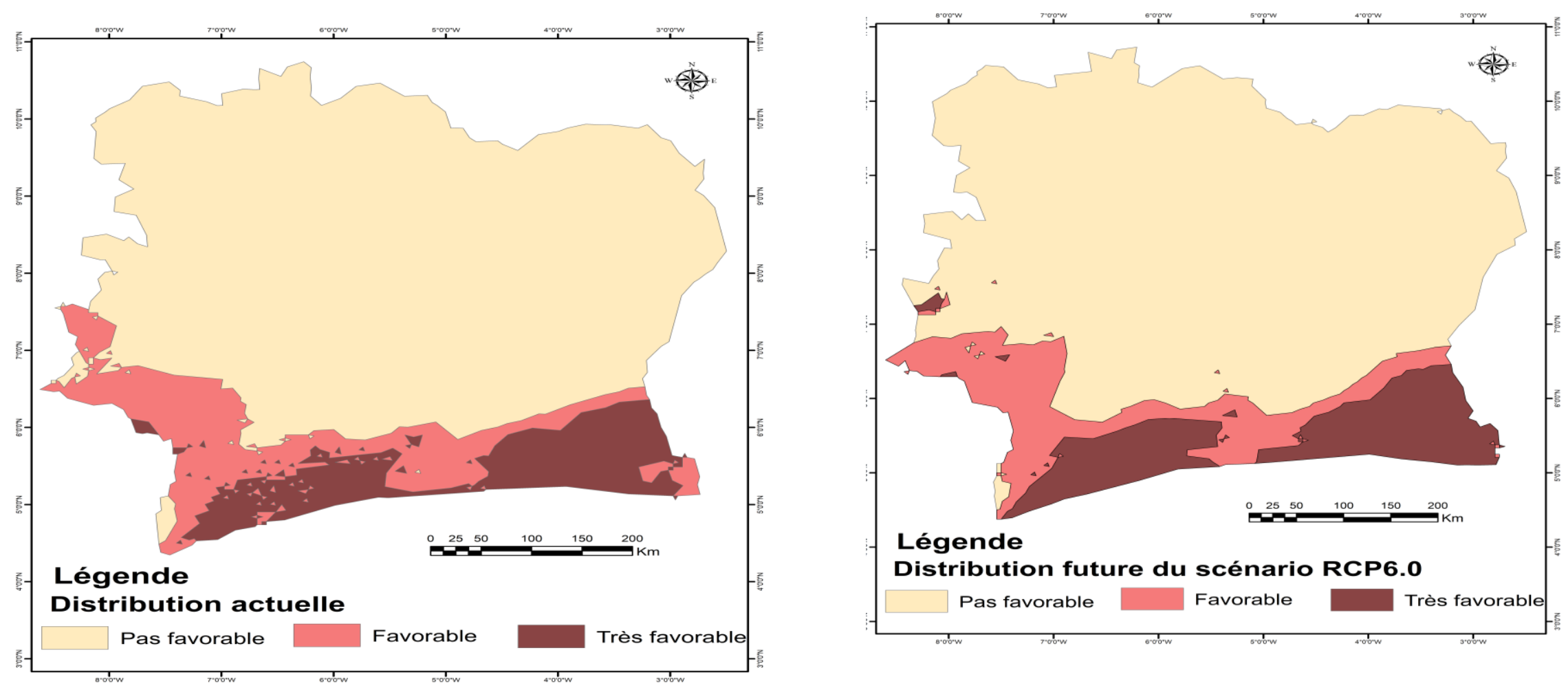

Figure 2 : Cartes de distribution des habitats actuels et futurs de Makoré dans les zones phytogéographiques en Côte d'Ivoire, à l'aide du scénario RCP 6.0 . 
Tableau 4 : Variation de la superficie et de la tendance de l'aire de distribution de Makoré en fonction des périodes, à l'aide du scénario RCP 8.5.

\begin{tabular}{lcccccc}
\hline & \multicolumn{5}{c}{ Aire de distribution } \\
\cline { 2 - 7 } & \multicolumn{2}{c}{ Zones non favorable } & \multicolumn{2}{c}{ Zones favorable } & \multicolumn{2}{c}{ Zones très favorables } \\
\cline { 2 - 7 } Périodes & $\begin{array}{c}\text { Superficie } \\
\left(\mathrm{Km}^{2}\right)\end{array}$ & $\begin{array}{c}\text { Tendance } \\
(\%)\end{array}$ & $\begin{array}{c}\text { Superficie } \\
\left(\mathrm{Km}^{2}\right)\end{array}$ & $\begin{array}{c}\text { Tendance } \\
(\%)\end{array}$ & $\begin{array}{c}\text { Superficie } \\
\left(\mathrm{Km}^{2}\right)\end{array}$ & $\begin{array}{c}\text { Tendance } \\
(\%)\end{array}$ \\
Actuelle & 249263 & 77,32 & 40741 & 12,70 & 32031 & 9,98 \\
$\begin{array}{l}\text { Future } \\
(\mathbf{2 0 5 0})\end{array}$ & 250413 & 77,68 & 35949 & 11,15 & 36005 & 11,17 \\
& & & $\mathbf{- 4 ~ 7 7 2}$ & $\mathbf{- 1 , 5 5}$ & $\mathbf{+ 3 ~ 9 7 4}$ & $\mathbf{+ 1 , 1 9}$ \\
\hline
\end{tabular}

Le signe (-) indique une perte d'habitat favorable et le signe (+) indique un gain

RCP : Representative Concentration Pathways (scénario élaboré pour stimuler l'ampleur des changements climatiques futurs).

Tableau 5: Valeurs des contributions des variables climatiques utilisées pour la modélisation de la distribution géographique de Makoré.

\begin{tabular}{|c|c|c|c|c|}
\hline Codes & Dénomination des variables bioclimatiques & $\begin{array}{l}\text { Période } \\
\text { actuelle }\end{array}$ & $\begin{array}{l}\text { Période future } \\
(2050)-\text { RCP } 6.0\end{array}$ & $\begin{array}{l}\text { Période future } \\
(2050) \text {-RCP } 8.5\end{array}$ \\
\hline $\mathrm{BIO} 2$ & $\begin{array}{l}\text { Étendue diurne moyenne (Moyenne } \\
\text { mensuelle } \\
\text { de (tmax - tmin) }\end{array}$ & & 13,6 & 10,7 \\
\hline $\mathrm{BIO} 3$ & Isothermalité (BIO2/BIO7) $(* 100)$ & 0,2 & & \\
\hline $\mathrm{BIO} 4$ & $\begin{array}{l}\text { Saisonnalité des températures (écart-type * } \\
100 \text { ) }\end{array}$ & 2,4 & 6,1 & 0,9 \\
\hline BIO5 & Température maximale du mois le plus chaud & 0,2 & 0,4 & \\
\hline BIO6 & Température minimale du mois le plus frais & 4,8 & 0,5 & 5,1 \\
\hline $\mathrm{BIO} 8$ & $\begin{array}{l}\text { Température moyenne du trimestre le plus } \\
\text { arrosé }\end{array}$ & 0,4 & & 0,2 \\
\hline BIO9 & $\begin{array}{l}\text { Température moyenne du trimestre le plus } \\
\text { sec }\end{array}$ & 4,5 & 3,0 & 2,3 \\
\hline $\mathrm{BIO} 12$ & Précipitations annuelles & 10,7 & 1,2 & 1,4 \\
\hline $\mathrm{BIO} 13$ & Précipitations du mois le plus arrosé & 9,1 & 14,7 & 24,7 \\
\hline $\mathrm{BIO} 14$ & Précipitations du mois le plus sec & 53,8 & 47,7 & 45,7 \\
\hline BIO15 & $\begin{array}{l}\text { Saisonnalité des précipitations (coefficient de } \\
\text { variation) }\end{array}$ & 1,5 & & 0,8 \\
\hline $\mathrm{BIO} 16$ & Précipitations du trimestre le plus arrosé & 12,3 & 10,9 & \\
\hline BIO18 & Précipitations du trimestre le plus chaud & & 0,3 & \\
\hline BIO19 & Précipitations du trimestre le plus frais & 0,2 & 4,6 & 8,3 \\
\hline
\end{tabular}



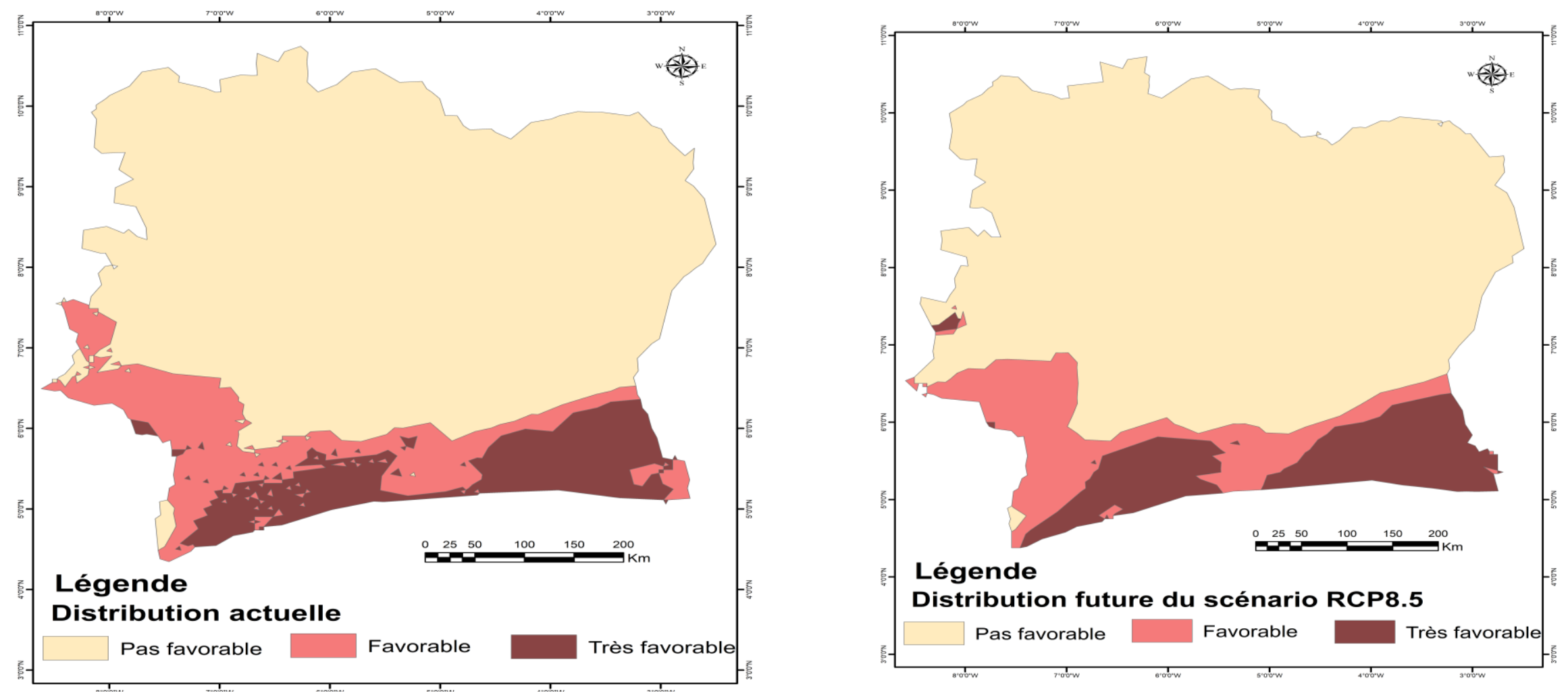

Figure 3 : Cartes de distribution des habitats actuels et futurs de Makoré dans les zones phytogéographiques en Côte d'Ivoire, à l'aide du scénario RCP 8.5 . 


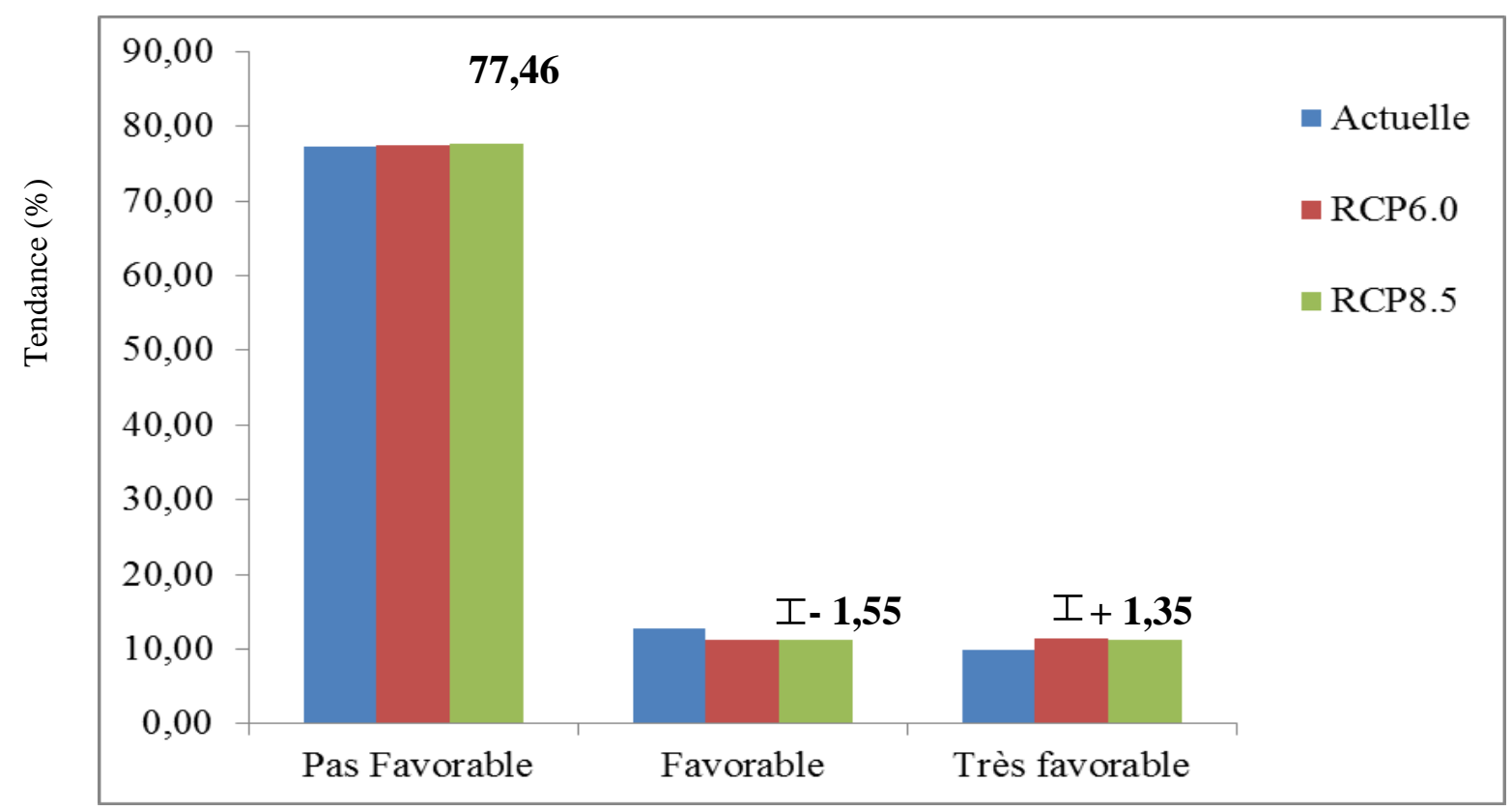

Types d'habitats

Figure 4 : Histogramme de l'évolution des tendances des habitats de Makoré en Côte d'Ivoire en fonction des périodes climatiques, à l'aide des scénarios.

\section{DISCUSSION}

\section{Aire de distribution potentiellement favorable au Makoré}

Les résultats de nos travaux montrent que les zones de distribution potentiellement favorables (actuelles et futur (horizon 2050)) de Makoré se retrouveront exclusivement dans la zone phytogéographique ombrophile. Cela témoigne du recule de l'aire de distribution de Makoré dans la forêt dense humide sempervirente et le littoral. En effet, selon Aké-Assi (2002) le Makoré est préférentiellement abondant dans la zone ombrophile de la forêt ivoirienne, mais des individus sont rencontrés dans la zone mésophile, notamment les forêts classées de Bossematié, du Haut-Sassandra, de Mopri, de Singrobo, de la Téné, etc. Par ailleurs, la majeure partie de la couverture végétale de la Côte d'Ivoire n'est pas favorable à la distribution de Makoré dans les conditions climatiques actuelles et futures (horizon 2050). Cela pourrait s'expliquer par le grand endémisme de Makoré pour les forêts ombrophiles. Ces habitats de Makoré sont probablement localisés majoritairement dans les aires protégées; notamment les parcs nationaux, les réserves biologiques et les forêts classées. C'est donc à juste titre que Houinato et al. (2001) ont mentionné que les aires protégées constituent des refuges qui favorisent encore la conservation de la biodiversité, notamment des espèces endémiques et internationalement ou localement en danger face aux effets du changement climatique à l'horizon 2050 . D'ailleurs, les résultats de cette étude sont en concordance avec ceux obtenus par Asseh (2019) et Asseh et al. (2019) qui ont montré que la distribution, selon les conditions climatiques actuelles et futures (horizon 2050) des espèces modèles des Acanthaceae se situe dans les aires protégées ou dans leurs périmètres. Cependant, les projections à l'horizon 2050 indiquent une régression de la superficie des habitats potentiellement "favorables" de Makoré. Cette régression s'expliquerait probablement par la projection 
de la hausse de la température et de la baisse du niveau des précipitations au niveau de la Côte d'ivoire, comme l'on signalé Kouakou et al. (2012) et Oga et al. (2016) au cours de leurs différents travaux. En effet, les résultats des travaux de Gbesso et al. (2013), ont déjà mis en évidence la régression des zones favorables à la distribution de certaines espèces. Ceci conforte l'hypothèse de WWF (2011), selon laquelle, les changements climatiques pourraient modifier l'aire de répartition des espèces. En effet, la hausse des températures et la baisse des précipitations seraient le résultat de l'accroissement des concentrations des rejets anthropiques de Gaz à Effet de Serre (GES) fortement liées à la régression des forêts denses sempervirentes. Cette régression des forêts constatés en Côte d'Ivoire à l'instar des pays de la zone tropicale est aussi imputable à la démographie galopante entrainant les besoins de terres et des pressions anthropiques qui occasionnent une perte des habitats naturels favorables aux espèces telles qu'observée dans nos résultats. $\mathrm{La}$ régression des zones de distribution potentiellement favorables des espèces se fait par conversion en zone potentiellement non favorables induisant un accroissement de leur superficie. Par contre, le gain des habitats "très favorables" à l'horizon 2050 indiquerait probablement que l'espèce restera confinée dans les forêts denses humides sempervirentes et le littoral pour accroitre ses chances de survie. En effet, dans cette zone phytogéographique, les perturbations climatiques même significatives ne semblent pas affecter considérablement la distribution de Makoré.

\section{Validation du processus de modélisation et contributions des variables bioclimatiques}

Les valeurs de l'AUC de plus de 0,90 indiquent une excellente qualité des modèles générés pour la distribution des habitats de Makoré. En effet ces valeurs qui varient entre 0,901 et 0,936 indiquent la robustesse de la modélisation effectuée et atteste de l'excellente performance de l'algorithme MaxEnt à prédire les différents habitats de Makoré. Les résultats de la présente étude sont en concordance avec ceux de Fandohan et al. (2015) sur la qualité du logiciel MaxEnt.
Selon l'auteur, le logiciel ne constitue pas de problèmes lorsque la zone d'étude couvre une échelle réduite et que l'aire de référence pour tester et calibrer le modèle est limitée aux conditions climatiques où l'espèce est sous l'influence des mêmes facteurs climatiques.

Concernant la contribution des variables environnementales, les résultats révèlent que les "Précipitations du mois le plus sec (Bio 14) » est la variable bioclimatique qui a présenté la meilleure contribution à la prédiction des modèles pour la distribution de Makoré à l'horizon 2050. L'importante contribution de cette variable signifie que la distribution future de Makoré sera principalement influencée par les variations de pluviométrie et des activités anthropiques.

\section{Conclusion}

Cette étude a permis de disposer des données sur les simulations de changements climatiques à l'horizon 2050 sur la distribution de Tieghemella heckelii (Sapotaceae) ou Makoré en Côte d'Ivoire. Il ressort que les zones potentiellement favorables à l'aire de distribution de Makoré seront, de façon générale, de l'ordre de $22,54 \%$ de superficie du territoire ivoirien. Ces habitats seront localisés exclusivement dans la zone phytogéographique ombrophile et s'étendront de l'Ouest montagneux à l'Est du pays, en passant par le Sud et le Littoral. Probablement ceux-ci seront principalement dans les aires protégées. Cependant, l'effet des variations climatiques et des activités anthropiques entraineront une réduction de superficies des zones potentiellement "favorables" à l'aire de distribution de Makoré, de l'ordre de 4775,5 $\mathrm{km}^{2}$, en moyenne, soit $1,55 \%$ pour les deux scénarios (RCP 6.0 et RCP 8.5). Par contre, un accroissement des zones potentiellement "très favorables", de l'ordre de $4484,5 \mathrm{~km}^{2}$ de superficie, en moyenne, soit $1,35 \%$ pour les deux scénarios seront observés. Par ailleurs, l'importante contribution de la variable bioclimatique «Précipitations du mois le plus sec (Bio 14) » signifie que la distribution future de Makoré sera principalement influencée par les variations de pluviométrie. Malgré ces perturbations qui seront observées 
sur l'aire de distribution de Makoré, les résultats montrent que les variations environnementales en Côte d'Ivoire demeureront favorables à la distribution de l'espèce, qui de loin est en danger d'extinction dans les forêts tropicales. Cette étude sur l'évaluation de la distribution spatiotemporelle de Makoré, en fonction de la prédiction des variations climatiques aidera certainement les gestionnaires du patrimoine forestier ivoirien dans leurs prises de décision sur la conservation et de la protection de la biodiversité.

\section{CONFLIT D'INTERETS}

Les auteurs déclarent qu'ils n'ont aucun conflit d'intérêts pour la publication de cet article.

\section{CONTRIBUTIONS DES AUTEURS}

SCD, auteur principal de cet article, a collecté les données d'occurrence de Makoré dans les trois importants herbiers de Côte d'Ivoire. Elle a défini le thème et les objectifs de cette étude et réaliser les différentes analyses. Elle a aussi participé à la rédaction de l'article. KK a supervisé la collecte des données et orienter la démarche méthodologique et les résultats obtenus. Il a aussi corrigé la forme et le fond du manuscrit proposé. HND a contribué significativement à la rédaction de cet article en participant aussi aux analyses de données et a réalisé les différentes cartes de distribution continues dans cet article.

\section{REMERCIEMENTS}

Nous exprimons notre reconnaissance aux Directeurs du Centre National de Floristique (CNF) de l'Université Félix HOUPHOUËT-BOIGNY (Côte d'Ivoire), de l'Institut Botanique Aké-Assi (IBAAN) et du Centre Suisse de Recherche Scientifique (CSRS), de nous avoir permis de collecter les données d'occurrences de Makoré dans leurs différents centres de recherche.

\section{REFERENCES}

Adou Yao CY. 2005. Pratiques paysannes et dynamique de la biodiversité dans la Forêt de Monogaga (Côte d'Ivoire). Thèse de Doctorat, Département
Hommes Natures Sociétés, Muséum national d'histoire naturelle, Paris, France, $233 \mathrm{p}$.

Aké-Assi L. 1997. Préface. In Bois de Côte d'Ivoire: Précis de Reconnaissance des Arbres Commercialisés, Sattler D (Ed.). CEDA : Abidjan, Côte d'Ivoire ; 7-9.

Aké-Assi L. 2002. Flore de la Côte d'Ivoire 2, Catalogue, Systématique, Biogéographie et Ecologie. Conservatoire et Jardin Botanique de Genève : Suisse Boisseria ; 58: $441 \mathrm{p}$.

Araújo MB, Pearson RG, Thuiller W, Erhard M. 2005. Validation of species climate impact models under climate change. Global Change Biology, 11: 1504 -1513. DOI:

https://doi.org/10.1111/j.13652486.2005. 01000.x

Asseh EE. 2019. Etudes floristique, phytogéographique et ethnobotanique des Acanthaceae de Côte d'Ivoire: cas de la réserve naturelle partielle de dahliafleur, dans le district autonome d'Abidjan et essai de domestication de Justicia flava (Forssk) Vahl. pour l'horticulture. Thèse de Doctorat Unique, UFR Biosciences, Université Félix Houphouët-Boigny, Abidjan, Côte d'Ivoire, $178 \mathrm{p}$.

Asseh EE, Ake-Assi E, Koffi KJ. 2019. Diversité biologique et influence des changements climatiques sur la distribution géographique de quelques espèces d'Acanthaceae en Côte d'Ivoire. International Journal of Biological and Chemical Sciences, 13(2): 676-692. DOI : 10.4314/ijbcs.v13i2.9

Brou YT. 2005. Climat, mutations socioécologiques et paysages en Côte d'Ivoire. Mémoire de synthèse des activités scientifique. Institut de Géographie Tropicale d'Abidjan, Université de Cocody-Abidjan, Abidjan, Côte d'Ivoire, $226 \mathrm{p}$.

Davo E. 2011. Contribution à l'évaluation de la biodiversité des espèces de faunes aviennes des collines. Mémoire de maitrise Département de Géographie et Aménagement du Territoire, Faculté des Lettres, Arts et Sciences Humaines, Université d'Abomey-Calavi, 85p. 
Elith J, Kearney M, Philips S. 2011. The art of modeling rangeshifting species. Methods in Ecology and Evolution 1: 330-342. DOI : 226360-1-10-330029 (1).pdf

Fandohan B, Gouwakinnou G, Fonton NH, Sinsin B, Liu J. 2013. Impact des changements climatiques sur la répartition géographique des aires favorables à la culture et à la conservation des fruitiers sous-utilisés : cas du tamarinier au Bénin. Biotechnol. Agron. Soc. Environ., 17(3): 450-462. DOI:

https://popups.uliege.be:443/17804507/i ndex.php?id=10186.

Gbesso FHG, Tente BHA, Gouwakinnou NG, Sinsin BA. 2013. Influence des changements climatiques sur la distribution géographique de Chrysophyllum albidum G. Don (Sapotaceae) au Benin. Int. J. Biol. Chem. Sci., 7(5): 2007-2018. DOI: http://dx.doi.org/10.4314/ijbcs.v7i5.18

GIEC. 2007. Groupe d'Experts Intergouvernemental sur l'Evolution du Climat, Bilan 2007 des changements climatiques. Contribution des Groupes de travail I, II et III au quatrième Rapport d'évaluation du Groupe d'experts intergouvernemental sur l'évolution du climat, Genève-Suisse, $103 \mathrm{p}$.

GIEC. 2013. Changements climatiques : les éléments scientifiques. Contribution du Groupe de travail I au 5ème rapport d'évaluation du GIEC, 34 p.

Gnagbo A, Kpangui KB, Adou YCY. 2015. Distribution des épiphytes de Côte d'Ivoire: effets des zones phytogéographiques et des variations pluviométriques. Afrique Science, 11(1): 175-186. DOI : $\quad 327518-1-10-$ 20150615(1).pdf

Heller N E et Zavaleta E S. 2009. Biodiversity management in the face of climate change: a review of 22 years of recommendations. Biological of Conservation, 142: 14-32. DOI : 10.1016/0006-3207/(94)00121-6

Houinato M, Sinsin B, Lejoly J. 2001. Impact des feux de brousse sur la dynamique des communautés végétales dans la forêt de
Bassila (Bénin). Acta Bot. Gall., 148(3): 237-251.

DOI:

10.1080/12538078.2001.10515891

IPCC. 2007. Climate Change. Synthesis Report. Contribution of Working Groups I, II \& III to the Fourth Assessment Report of the Intergovernmental Panel on Climate Change. Geneva Cambridge University Press Cambridge: UK and New York-USA; 339-378. DOI: 10.1017/CBO9780511546013.

Konaté S, Linsenmair KE. 2010. Diversité écologique de l'Afrique de l'Ouest: Importance, menaces et valorisation. In Atlas de la Biodiversité de l'Ouest: Côte d'Ivoire (Tome III), Konaté S, Kampmann D (eds). BIOTA : Abidjan \& Frankfurt/Main; 526 p.

Konan D, Bakayoko A, Tra Bi FH, Bitignon BGA, Piba SC. 2015. Dynamisme de la Structure diamétrique du peuplement ligneux des différents biotopes de la forêt classée de Yapo-Abbé, Sud de la Côte d'Ivoire. Journal of Applied Biosciences, 94: 8869-8879. DOI : 10.4314/jab.v94i1.10

Kouadio K. 2007. Etudes de la flore, de la végétation et de l'impact de l'éclaircie sélective par dévitalisation, sur les essences principales de la forêt classée de Bossematié, Est de la Côte d'Ivoire. Thèse Unique, UFR Biosciences, Université de Cocody-Abidjan, Côte d'Ivoire, p. 192.

Kouadio K, Kouassi KE, Dibi NH. 2012. Effet de l'éclaircie sélective sur la dynamique de la végétation arborescente dans la forêt classée de Bossematié (Côte d'Ivoire). Agron. Afr., 24(3): 219-230. DOI : 214952-1-10-20130330(1).pdf

Kouakou E, Goula BTA, Kouassi AM. 2012. Analyze of climate variability and change impacts on hydro-climate parameters: case study of Côte d'Ivoire. International Journal of Scientific \& Engineering Research, 3(2): 1-8. DOI: dx.doi.org/10.14299/ijser.2012.03.

Kouakou DB. 2014. Document de stratégie du programme national changement climatique (2015 - 2020), 84p.

Kouamé YM, Soro GE, Kouakou KE, Kouadio ZA, N'Diaye MEH, Goula 
BTA, Issiaka S. 2014. Scenarios des changements climatiques pour les précipitations et les températures en Afrique subsaharienne tropicale humide : cas du bassin versant de Davo, Côte d'Ivoire. Larhyss Journal, 18: 197-213. DOI : 214-668-1-PB.pdf

Liu C, White M, Newell G. 2013. Selecting thresholds for the prediction of species occurrence with presence-only data. Journal of Biogeography, 40: 778-789. DOI : https://doi.org/10.2307/2845584.

Manfo DA, Tchindjang M, Youta HJ. 2015. Systèmes agroforestières et conservation de la biodiversité dans un milieu fortement anthropisé : le cas d'Obala. Revue Scientifique et Technique Forêt et Environnement du Bassin du Congo, 5 : 22-34. DOI : 57-226-1-PB.pdf

Oga YMS, Adja M, Yapi AF, Kpan JG, Baka D, Yao KT, Biémi J. 2016. Projection de la variabilité climatique à l'horizon 2050 dans la zone côtière au Sud-Est de la Côte d'Ivoire (d'Abidjan à Aboisso). Larhyss Journal, 25: 67-81. DOI : 3601240-1-PB.pdf

Phillips SJ, Anderson RP, Schapire RE. 2006. Maximum entropy modeling of species geographic distributions. Ecol. Model. 190: 231-259. DOI: 10.1016/j.ecolmodel.2005.03.026

Sala E, Ballesteros E, Starr RM. 2001. Rapid decline of Nassau grouper spawning aggregations in Belize: Fishery Management and Conservation Needs, Fisheries, 26(10): 23-30. DOI: 10.1577/1548-8446(2001)026<0023: RDONGS>2.0.CO; 2.

Sorokoby VM, Saley MB, Kouamé KF, Djagoua EMV, Affian K, Biemi J. 2013.
Variabilité spatio-temporelle des paramètres climatiques et son incidence sur le tarrissement dans les bassins versants du Bô et Debo (département de Soubré au Sud-Ouest de la Côte d'Ivoire). International Journal of Innovation and Applied Studies, 2(3): 287-299. DOI : IJIAS-13-029-01.pdf

Toyi MS, Barima YSS, Mama A, André M, Bastin J-F, De Cannière C, Sinsin B, Bogaert J. 2013. Tree plantation will not compensate natural woody vegetation cover loss in the Atlantic Department of Southern Benin. Tropicultura, 31(1): 6270. DOI : http://www.tropicultura.org/text/v31n1/6 2.pdf

Tuo FN, Kouao JK, Kouassi FA, Kone M, Bakayoko A, Bogaert J. 2017. Etude de la diversité, de l'endémisme et de la distribution spatiale des Rubiaceae de Côte d'Ivoire. Int. J. Biol. Chem. Sci., 11(2): $\quad 777-797 . \quad$ DOI: https://dx.doi.org/10.4314/ijbcs.v11i2.20

Vroh BTA, Adou YCY, Kpangui KB, Goné BZB, Kouamé D, Koffi JK. 2016. Comparing Suitable Habitat Models to Predict Rare and Endemic Plant Species Distributions : What are the Limits of the Niche of Cola lorougnonis (Malvaceae) in Côte d'Ivoire ? Environment and Natural Resources Research, 6(3): 1-17. DOI:10.5539/enrr.v6n3p1

WWF (World wide fund for nature). 2011. Rapport des impacts du changement climatique sur les espèces. WWF, 21p.

UICN. 2019. IUCN Red List of Threatened Species.http://www.iucnredlist.org. visité le 15 Novembre 2020. 